EPJ Web of Conferences 77, 00010 (2014)

DOI: $10.1051 /$ epjconf / 20147700010

(C) Owned by the authors, published by EDP Sciences, 2014

\title{
Measurement of air flow in duct by velocity measurements
}

\author{
Isabelle Caré ${ }^{1, a}$, Francis Bonthoux ${ }^{2}$ and Jean-Raymond Fontaine ${ }^{2}$ \\ ${ }^{1}$ CETIAT, Domaine scientifique de la Doua, 25 avenue des Arts, 69100 Villeurbanne, France \\ ${ }^{2}$ INRS, Département Ingénierie des Procédés, 1 rue du Morvan, 54519 Vandœuvre-lès-Nancy, France
}

\begin{abstract}
To measure a flow in a closed duct, one of the available methods is to explore the velocity field. With this method, the quality of the flow measurement is very dependent on the location of the velocity measurement points in the duct section. Recommendations about velocity schemes are proposed in international standards (ISO 3966, ISO 7145, EN 12599 ...) for circular and rectangular ducts. These recommendations assume that a turbulent flow profile is established. This requires flow profiler and/or long straight lengths upstream and downstream the measurement section. On site, these recommendations are difficult to apply strictly because conditions of straight lengths are often not available. Secondly, the velocity measurement schemes proposed in standards are often time consuming and users prefer sometimes to simplify them. In this case, the estimation of the measurement error is not known. A numerical study has been carried out to investigate the influence of the velocity measurement scheme on the flow measurement when the distance between disturbances and the measurement section is small in the case of circular and rectangular ducts. The results are presented in term of measurement error according to the shape of the duct, velocity scheme, number of velocity measurements, distance between disturbances and measurement section.
\end{abstract}

\section{Introduction}

To determine the flow rate in a closed duct, one of the common way is to explore the velocity field. The principle of this technique is to divide the duct cross-section in elementary surface elements, to measure the velocity in each of these elements and to calculate a average velocity weighted over the surfaces. The flow rate is then this mean velocity multiplied by the duct cross-section.

This method needs a lot of measurement points as well as a turbulent velocity profile to allow a sufficient accuracy level.

Some international standards propose different velocity exploration schemes, assuming assumptions on the velocity profile.

In ISO 3966 [1], the use of such method, known as Log-Tchebycheff method, needs a minimum of 12 measurement points distributed over 2 diameters for a circular duct and 25 measurement points for a rectangular duct. The assumption of a well-established turbulent velocity profile implies a minimum distance from any disturbances, which is 20 times the diameter (20D) in the case of a circular duct and 30D in the case of a rectangular one. If these conditions are met, the standard estimates a measurement uncertainty of $3 \%$ of the flow rate if a Pitot tube is used for velocity measurements. However, this method is rather time consuming. On the opposite, the NF X 10-113 standard [2] proposes a single-point

\footnotetext{
${ }^{\mathrm{a}}$ Corresponding author: isabelle.care @ cetiat.fr
}

measurement method in circular ducts,. Two different positions are proposed, at the centre of the duct or at a distance of $0.121 \mathrm{D}$ from the wall. The counterpart of this interesting time-saving method is the minimum distance from any disturbances which increases up to 50D when the measurement is performed at the centre of the duct and at $80 \mathrm{D}$ for the $0.121 \mathrm{D}$ position in a circular duct.

Some other standards [3, 4] propose also alternative exploration schemes.

Practical ventilation systems have rarely straight lengths to comply with the conditions described in the standards. Secondly, the time available for the personnel in charge of the measurements is often not compatible with the implementation of the standardized methods.

It is then of great interest to evaluate the maximum error which can be made if all such conditions are not met, i.e. the straight lengths are shorter than those that are recommended or the number of measuring points is reduced.

\section{Methodology}

Bonthoux et al. [5] have already experimentally investigated the effect of reduced straight lengths and exploration schemes on the maximum error which can occur after a disturbance in the case of a circular duct. According to the number of traverses, the number of measuring points by traverse, the straight length of the duct 
and its diameter, Bonthoux et al. proposed an equation to evaluate the maximum error which can occur whatever the disturbance is.

To extend the analysis carried out in [5] to rectangular ducts, numerical simulation has been performed. A CFD code is, indeed, a good tool to explore - by simulation - a lot of configurations.

As a first step of validation, numerical results have been compared to experimental data from [5] for circular duct. Then, additional tests for rectangular ducts were made to complete the study.

\subsection{Description of the numerical tool}

The great advantage of a numerical tool is the ease to vary the test parameters as compared to experiments.

In recent years, increasing capabilities of computers and development of more and more precise physical models have enabled the CFD code to become a reliable engineering tool.

The computational tool used for the present work is PHOENICS by CHAM [6]. It is a multi-purpose Computational Fluid Dynamics (CFD) software package which is applicable to steady or unsteady, one-, two- or three-dimensional, turbulent or laminar, multi-phase, compressible or incompressible flows using Cartesian, cylindrical-polar or curvilinear coordinates. The code is based on a finite-volume approach and includes models, enabling the simulation of a wide range of situations.

In the present work, calculations were performed using an iterative integration procedure of the finite volume equations.

Navier-Stokes equations together with the continuity equation make a closed set of equations, the solution of which provides a description of laminar and turbulent flows. In principle, it is possible to simulate any turbulent flow by exactly solving the Navier-Stokes equations, as far as the boundary conditions are correctly set. In practice, all the details of the motion are often not needed and only averaged parameters are required. To simplify the solution of Navier-Stokes equations, turbulence models are often used to describe the turbulent correlations. LVEL turbulence model were chosen as a good compromise between quality and calculation time. LVEL model is a feature of PHOENICS, introduced in 1993. It calculated the turbulent viscosity used in the turbulence models using the Spalding's law of the wall. This model is particularly suitable for cases where the flow is constraint with many walls where the grid cannot be refined enough for more usual turbulence models, such as k- $\varepsilon$ turbulence model.

\subsection{Comparison with experimental results}

Some preliminary calculations at different mean velocities and duct diameter showed that the velocity profiles were always the same, as far as the flow is turbulent. All the results are then presented dimensionless.

Bonthoux et al. investigated the velocity field at 1D, $4 \mathrm{D}, 10 \mathrm{D}$ and $45 \mathrm{D}$ of various disturbances, as shown in Figure 1 below.
Figure 1. Disturbances tested by Bonthoux et al [5]

\begin{tabular}{|l|l|}
\hline Straight pipe inlet & \\
\hline Elbow & co-planar \\
\hline 2 non-co-planar elbows & \\
\hline Y junction & \\
\hline Y junction with reducer & \\
\hline Open damper & \\
\hline Closed damper (flap at $30^{\circ}$ ) \\
\hline
\end{tabular}

The study in [5] has shown that, among all the tested disturbances, the elbows (single, 2 co-planar and 2 non coplanar) are those which show the largest velocity profile distortion. Thus, it has been decided to perform the numerical simulations of ducts presenting only these kinds of disturbances.

Figure 2 presents a comparison of the experimental and calculated velocity profiles for two relevant configurations.

Figure 2. Comparison of the experimental and calculated

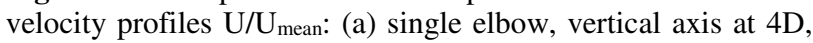
(b) 2 co-planar elbows, horizontal axis at 10

(a)

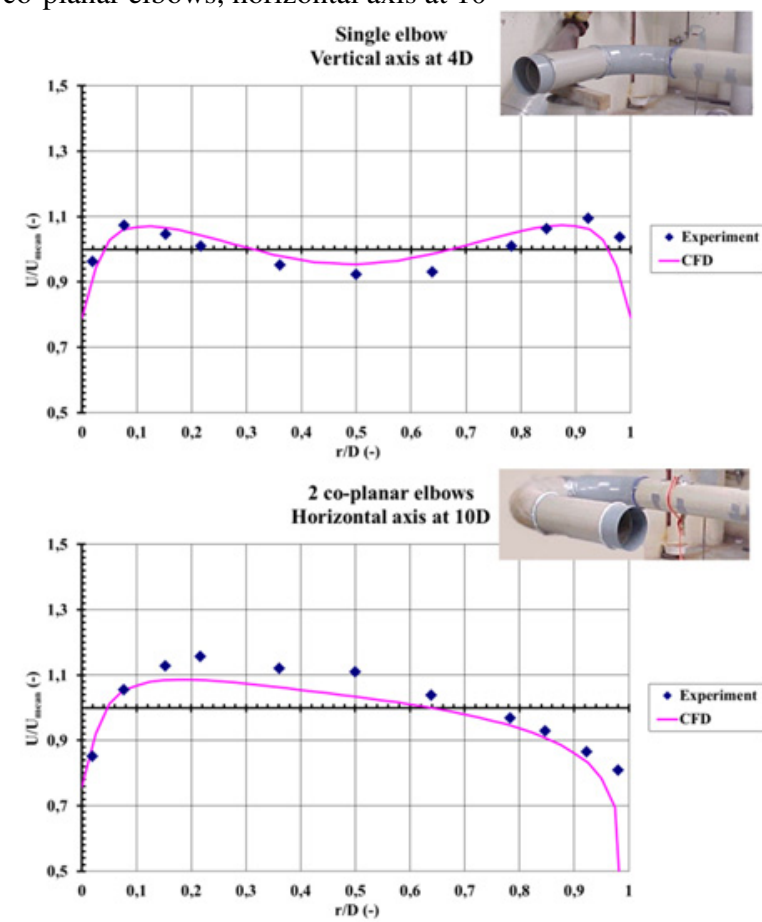

The calculations are in good agreement with the experiments with respect of the velocity profile.

Figure 3 shows the deviation between the experimental and calculated mean velocities along horizontal and 
vertical axes. The mean velocities are calculated along a diameter.

Figure 3. Comparison of the experimental and calculated mean velocities: (a) horizontal axis, (b) vertical axis

(a)

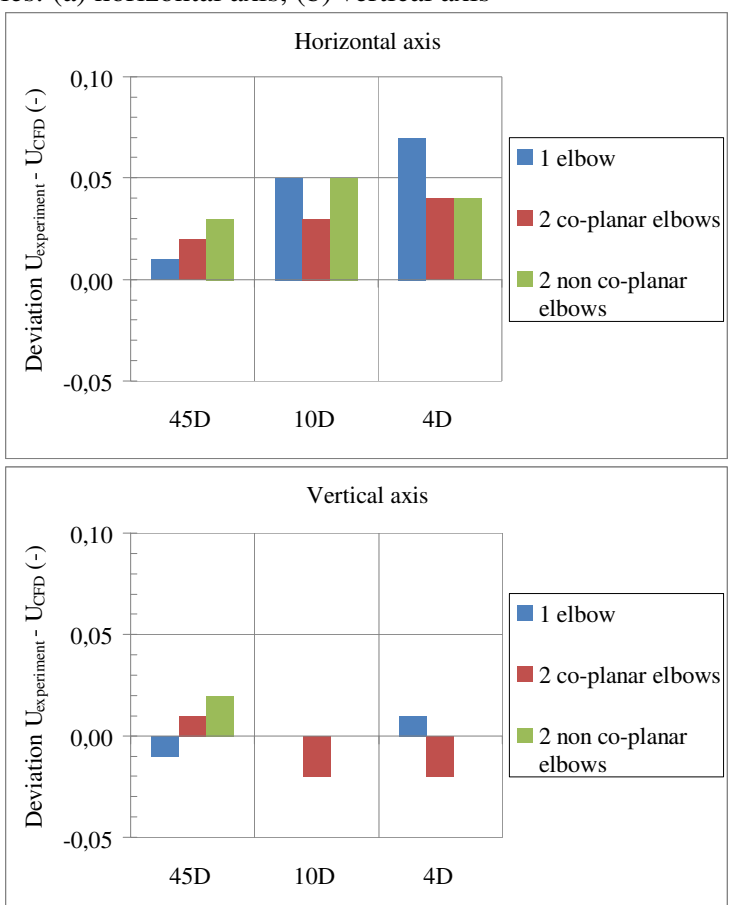

The deviation between the mean and the calculated velocity is rather small and acceptable if the measurement uncertainty of the experiments and of the simulations are taken into account.

The use of such CFD code is then considered as validated for the purpose of this study.

\section{Results}

The aim of the present work is to establish the order of magnitude of the error which can occur either when the conditions defined by the standards are not fulfilled or when non-standardised methods are applied for the determination of the flow rate in a duct by exploration of its velocity profile.

\subsection{Circular ducts}

The experimental investigations in [5] were carried out with a $200-\mathrm{mm}$ inner diameter pipe at a Reynolds number around 200000. Twenty-one experimental points were measured by means of a Pitot tube associated with a micromanometer at a distance 1D, 4D, 10D and 45D from the disturbances presented in Figure 1: two perpendicular 10-point traverses according to a "log-linear" scheme and one additional point at the centre were selected.

The average velocity is determined in each plane, $\mathrm{U}_{\mathrm{XD}}$, as the arithmetic mean of all the velocities measured in that plane. It is compared with the calculated one at $45 \mathrm{D}$, namely $\mathrm{U}_{45 \mathrm{D}}$. This plane is, indeed, considered as the reference one, in which the turbulent velocity profile is well established. The absolute error at a distance XD from a disturbance is then calculated with the following equation:

$$
|E|=\left|1-\frac{U_{X D}}{U_{45 D}}\right|
$$

The influence of some parameters were also evaluated by Bonthoux et al. . As an example, the Figure 4 below shows the absolute error against the dimensionless distance from disturbances.

Figure 4. The absolute error as a function of L/D [from ref. 5] |E् (\%)

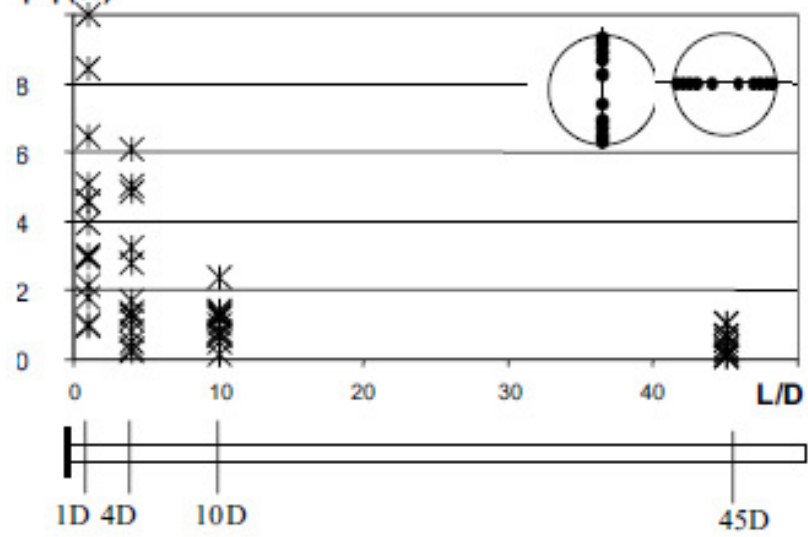

Combining the effect of the different parameters, Bonthoux et al. estimated the maximum absolute error as a function of the number of measurement points, the number of traverses and the dimensionless distance from a disturbance, whatever are the disturbances:

$$
E=0.7 \times \frac{1}{\left(\frac{L}{D}\right)^{0.7}} \times \frac{1}{k} \times \frac{1}{6 \times\left(1-e^{-p / 6}\right)}
$$

where:

$E$ is the maximum absolute error in \%,

$k$ is the number of traverses,

$p$ is the number of points per traverse,

$L$ is the straight length from disturbance,

$D$ is the duct diameter

This equation has been used to generate a table in which the maximum error is calculated according to the value of the different parameters, $k, p$ and $L / D$ (see Table 1). 
Table 1. Maximum calculated error

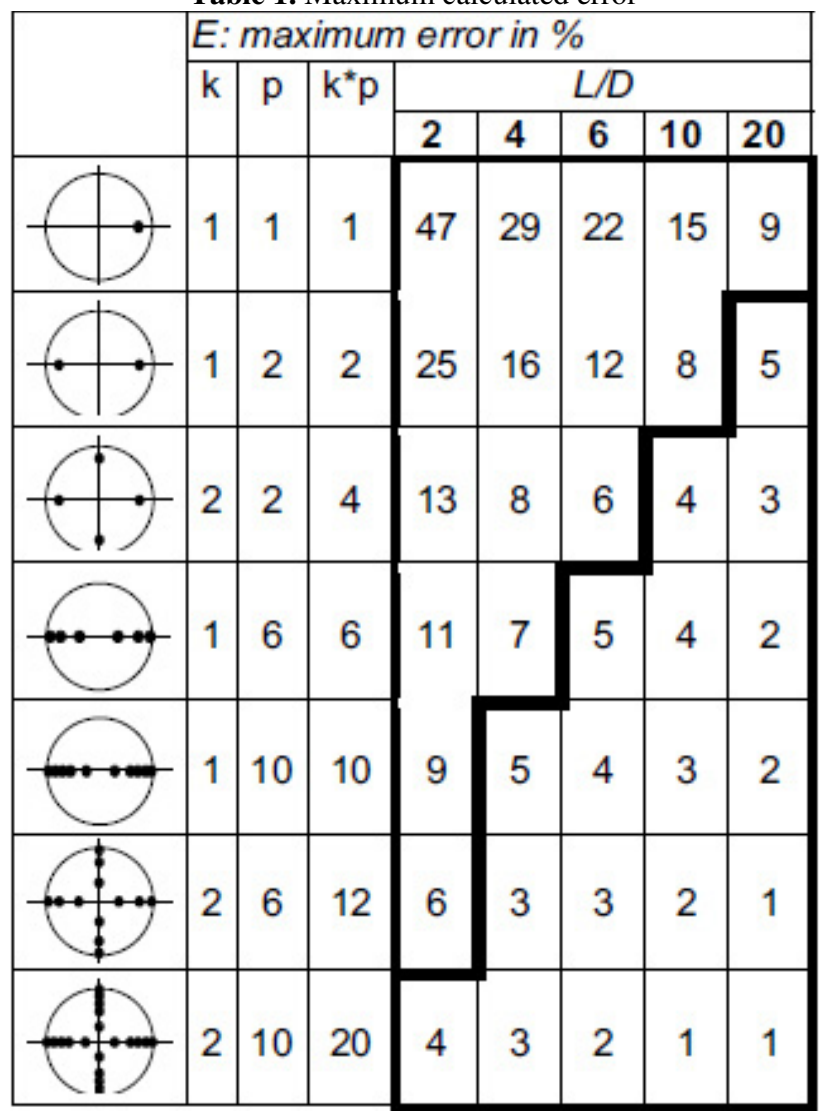

The above Table 1 provides an operational tool to choose the points scheme to be applied according to the distance from disturbances and the expected maximum error.

\subsection{Rectangular ducts}

The influence of the same parameters was studied for the rectangular ducts as well, i.e. the number of traverses, the number of measuring points per traverse and the dimensionless distance from disturbances, $L / D$ were considered. $L / D$ was evaluated by using the hydraulic diameter calculated with the following equation:

$$
D=S / P
$$

where

$S$ is the section of the duct

$P$ is the perimeter of the duct

For a rectangular duct, this equation can be written as follows:

$$
D=\frac{a \times b}{2 \times(a+b)}
$$

where:

$a$ is the width of the rectangular duct

$b$ is the height of the rectangular duct.

This study was fully carried out numerically for ducts with a single elbow, 2 co-planar elbows and 2 non coplanar elbows.

The results of the calculations were analysed to calculate the mean velocity at different distance from distrubances according to different methods of exploration of the velocity field.
The methods which were considered here are those of ISO 3966 [1], known as Log-Tchebycheff method, EN 12599 [3] and Pr EN 16211 [4]. These two last standards were included because they are concerning on site measurements of air flows in buildings ventilation. They propose alternative methods to ISO 3966.

In practice, some non-standardised methods are often used on site because of a lack of knowledge and/or time by technical personnel. The most popular approach is to measure the velocity at 5 different locations along one or three lines according to the shape of the duct. Such a shape is described with a "shape factor" defined as the ratio between the height and the width of the duct, $b / a$. Two cases are generally considered according to the shape factor value (close to unity or far from unity). If the ratio is far from to 1 , the duct has a high aspect ratio (a "flat shape") and the flow distortion due to the disturbances is high. It can then be difficult to strictly follow the exploration schemes given by the standards because of the available space inside the duct.

The numerical results were gathered together in such a way to be convenient to a user. Table 2 and Table 3 present the case of the rectangular duct with a shape factor equal to 1 and 4, respectively. According to the exploration scheme of the velocity field and number of traverses, the maximum absolute error (Eq. (1)) was calculated.

Table 2. Expected maximum error using t method by exploration of flow field in the case of a rectangular duct with a

\begin{tabular}{|c|c|c|c|c|}
\hline \multirow[t]{2}{*}{ Exploration scheme } & \multirow[t]{2}{*}{$\begin{array}{c}\text { Number of } \\
\text { traverses } \\
\text { (points) }\end{array}$} & \multicolumn{3}{|c|}{$\begin{array}{c}\text { Maximum error (\%) } \\
\text { L/D : Upstream } \\
\text { distance from } \\
\text { disturbances }\end{array}$} \\
\hline & & 5 & 10 & 45 \\
\hline \multirow{2}{*}{ 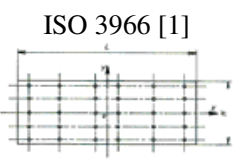 } & $5(25)$ & 5 & 3 & 1 \\
\hline & $1(5)$ & 39 & 9 & 8 \\
\hline \multirow{2}{*}{$\begin{array}{l}\text { EN 12599 [3] } \\
: \bullet:\end{array}$} & $3(9)$ & 11 & 10 & 8 \\
\hline & $1(3)$ & 38 & 26 & 19 \\
\hline \multirow{2}{*}{ 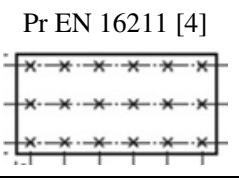 } & $3(18)$ & 5 & 3 & 2 \\
\hline & $1(6)$ & 28 & 9 & 8 \\
\hline \multirow{2}{*}{$\begin{array}{c}\text { Non standardised } \\
\text { method } \\
\vdots\end{array}$} & $2(9)$ & 14 & 16 & 10 \\
\hline & $1(5)$ & 62 & 25 & 17 \\
\hline
\end{tabular}
shape factor close to 1 
Table 3. Expected maximum error using a method by exploration of flow field in the case of a rectangular duct with a shape factor equal to 4

\begin{tabular}{|c|c|c|c|c|}
\hline \multirow{3}{*}{ Exploration scheme } & \multirow{3}{*}{$\begin{array}{l}\text { Number of } \\
\text { traverses } \\
\text { (points) }\end{array}$} & \multirow{2}{*}{\multicolumn{3}{|c|}{$\begin{array}{c}\text { Maximum error (\%) } \\
\text { L/D : Upstream } \\
\text { distance from } \\
\text { disturbances }\end{array}$}} \\
\hline & & & & \\
\hline & & 5 & 10 & 45 \\
\hline \multirow{3}{*}{ 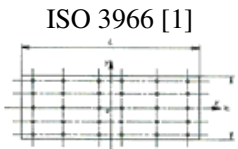 } & $5(25)$ & 4 & 4 & 2 \\
\hline & & & & \\
\hline & $1(5)$ & 10 & 7 & 7 \\
\hline \multirow{2}{*}{ EN 12599 [3] } & $3(9)$ & 8 & 8 & 5 \\
\hline & $1(3)$ & 17 & 11 & 9 \\
\hline \multirow[t]{2}{*}{ Pr EN 16211 [4] } & \multirow[b]{2}{*}{$1(6)$} & \multirow[b]{2}{*}{15} & \multirow[b]{2}{*}{6} & \multirow[b]{2}{*}{4} \\
\hline & & & & \\
\hline $\begin{array}{l}\text { Non standardised } \\
\text { method }\end{array}$ & \multirow{2}{*}{$1(5)$} & \multirow{2}{*}{15} & \multirow{2}{*}{7} & \multirow{2}{*}{7} \\
\hline - to-00-00- & & & & \\
\hline
\end{tabular}

The results obtained when the methods proposed by the standards are applied are of the same order of magnitude. As expected, by increasing the number of measuring velocity points, the expected maximum error reduces. As a consequence, the results obtained with the nonstandardised method are not satisfactory even at 45D upstream distance from disturbances. These methods should then not be recommended.

The magnitude of the maximum expected error is higher in the case of a rectangular duct (Table 2 and Table 3 ) than in a case of a circular one (Table 1), even if we cannot strictly compare the results because the exploration schemes are not the same. In particular, in the case of small straight lengths downstream the disturbances, i.e. smaller than $10 \mathrm{D}$, it could be difficult to achieve satisfactory uncertainty measurement in the case of a rectangular duct without spending a significant amount of time for a large number of measurement points.

\section{Conclusion}

The numerical work presented here can be considered as an extension of the experimental work carried out by Bonthoux et al. [5].

After the model validation, the influence of the distance from disturbances on the calculated flow rate was explored for different schemes of velocity field exploration. The results were then gathered in tables presenting the maximum expected error whatever the disturbance is. This approach can be exploited to estimate the uncertainty due to the exploration method.

To calculate the measurement uncertainty on the flow rate, additional components should be added such as:

- the uncertainty in the velocity measurement due to the instrument itself (calibration, resolution, drift, ...)
- the knowledge of the inner duct section

- the uncertainty in the positioning of the instrument inside the duct. This component can be significant in some cases. Precautions must be taken to minimize this uncertainty component.

These three additional components have to be considered carefully, though they can be difficult to estimate in critical on site configurations.

All the presented results and others were summarised in a Good Practice Guide for on site air flow measurement. This Guide in French is available at the CETIAT Web site as a free download.

\section{Acknowledgments}

ALDES and CIAT companies are thanked for their active contribution to the work presented here. The DGCIS service of the Ministry for Industrial Renewal is thanked for its financial support.

\section{References}

1. ISO 3966: 2008, Measurement of fluid flow in closed conduits. velocity area method for regular flows using Pitot static tubes

2 NF X 10-113: 1982, Détermination du débit des fluides dans les conduites fermées de section circulaire Méthode par mesure de la vitesse en un seul point

3. NF EN 12599: 2000, Ventilation des bâtiments. Procédures d'essai et méthodes de mesure pour la réception des installations de ventilation et de climatisation installées, (2000)

4. Pr EN 16211, Ventilation for buildings Measurement of air flows on site -methods, (to be published)

5. F. Bonthoux, J.-R. Fontaine, Measurement of flow rate in a duct by investigation of the velocity field. Uncertainty linked to the position and number of measurement points, Roomvent, (2002)

6. PHOENICS, CHAM, TR326, Phoenics VR Reference Guide (2010) 